JOURNAL OF THE

AMERICAN MATHEMATICAL SOCIETY

Volume 15, Number 2, Pages 273-294

S 0894-0347(01)00384-8

Article electronically published on December 20, 2001

\title{
TOWARDS THE AMPLE CONE OF $\bar{M}_{g, n}$
}

\author{
ANGELA GIBNEY, SEAN KEEL, AND IAN MORRISON
}

To Bill Fulton on his sixtieth birthday

\section{§0. IntroduCtion AND STATEMENT OF RESUlts}

The moduli space of stable curves is among the most studied objects in algebraic geometry. Nonetheless, its birational geometry remains largely a mystery and most Mori theoretic problems in the area are entirely open. Here we consider one of the most basic:

(0.1) Question (Mumford). What are the ample divisors on $\bar{M}_{g, n}$ ?

There is a stratification of $\bar{M}_{g, n}$ by topological type where the codimension $k$ strata are the irreducible components of the locus parameterizing pointed curves with at least $k$ singular points. An ample divisor must intersect any one dimensional stratum positively. It is thus natural to consider the following conjecture.

(0.2) Conjecture: $F_{1}\left(\bar{M}_{g, n}\right)$. A divisor on $\bar{M}_{g, n}$ is ample iff it has positive intersection with all 1-dimensional strata. In other words, any effective curve in $\bar{M}_{g, n}$ is numerically equivalent to an effective combination of 1-strata. Said differently still, every extremal ray of the Mori cone of effective curves $\overline{N E}_{1}\left(\bar{M}_{g, n}\right)$ is generated by a one dimensional stratum.

$F_{1}\left(\bar{M}_{0, n}\right)$ was previously conjectured by Fulton, whence our choice of notation. Special cases of $F_{1}\left(\bar{M}_{0, n}\right)$ are known (see KeelMcKernan96, §7) but the general case remains open. The central observation of this work is that the case of higher genus is no harder. Indeed, $\overline{N E}_{1}\left(\bar{M}_{g, n}\right)$ is naturally a quotient of $\overline{N E}_{1}\left(\bar{M}_{0,2 g+n}\right) \times$ $\mathbb{R}^{\geq 0}$ and Conjecture (0.2) is true for all $g$ iff it is true for $g=0$; see $(0.3)-(0.5)$. Further, the second formulation of Conjecture (0.2) holds except possibly for very degenerate families in $\bar{M}_{g, n}$; see (0.6). Moreover, we are able to give strong results on the contractions of $\bar{M}_{g, n}$ (i.e. morphisms with geometrically connected fibers from it to other projective varieties). For example we show that for $g \geq 2$ the only fibrations of $\bar{M}_{g, n}$ are compositions of a tautological fibration, given by dropping

Received by the editors September 5, 2000.

2000 Mathematics Subject Classification. Primary 14H10, 14 E99.

Key words and phrases. Ample cone, Mori cone, moduli space, stable curve.

During this research, the first two authors received partial support from a Big XII faculty research grant, and a grant from the Texas Higher Education Coordinating Board.

The first author also received partial support from the Clay Mathematics Institute, and the second from the NSF.

The third author's research was partially supported by a Fordham University Faculty Fellowship and by grants from the Centre de Recerca Matemática for a stay in Barcelona and from the Consiglio Nazionale di Ricerche for stays in Pisa and Genova.

(C)2001 American Mathematical Society 
points, with a birational morphism; see (0.9)-(0.11). Here are precise statements of our results:

We work over an algebraically closed field of any characteristic other than 2 (this last assumption is used only at the end of the proof of (3.5)).

By the locus of flag curves, we shall mean the image $\bar{F}_{g, n}$ of the morphism $f: \bar{M}_{0, g+n} / S_{g} \rightarrow \bar{M}_{g, n}$ induced by gluing on $g$ copies of the pointed rational elliptic curve at $g$ points (which the symmetric group $S_{g} \subset S_{g+n}$ permutes). The map $f$ is the normalization of $\bar{F}_{g, n}$.

(0.3) Theorem. $g \geq 1$. A divisor $D \in \operatorname{Pic}\left(\bar{M}_{g, n}\right)$ is nef iff $D$ has non-negative intersection with all the one dimensional strata and $\left.D\right|_{F_{g, n}}$ is nef. In particular, $F_{1}\left(\bar{M}_{0, g+n} / S_{g}\right)$ implies $F_{1}\left(\bar{M}_{g, n}\right)$.

Using the results of [KeelMcKernan96, Theorem (0.3) has the following consequence.

(0.4) Corollary. $F_{1}\left(\bar{M}_{g, n}\right)$ holds in characteristic zero as long as $g+n \leq 7$ and when $n=0$ for $g \leq 11$.

For $g \leq 4, F_{1}\left(\bar{M}_{g}\right)$ was obtained previously by Faber.

We call $E \subset \bar{M}_{g, n}(g \geq 1)$ a family of elliptic tails if it is the image of the map $\bar{M}_{1,1} \rightarrow \bar{M}_{g, n}$ obtained by attaching a fixed $n+1$-pointed curve of genus $g-1$ to the moving pointed elliptic curve. Any two families of elliptic tails are numerically equivalent. In (2.2), we will see that all the 1-strata except for $E$ are numerically equivalent to families of rational curves. Note that we abuse language and refer to a curve as rational if all of its irreducible components are rational. Thus it is immediate from (0.3) that the Mori cone is generated by $E$ together with curves in $\bar{R}_{g, n} \subset \bar{M}_{g, n}$, the locus of rational curves. The locus $\bar{R}_{g, n}$ is itself (up to normalization) a quotient of $\bar{M}_{0,2 g+n}$ : By deformation theory $\bar{R}_{g, n}$ is the closure of the locus of irreducible $g$-nodal (necessarily rational) curves. The normalization of such a curve is a smooth rational curve with $2 g+n$ marked points. Thus, if $G \subset S_{2 g}$ is the subgroup of permutations commuting with the product of $g$ transpositions $(12)(34) \ldots(2 g-12 g)$, the normalization of $\bar{R}_{g, n}$ is naturally identified with $\bar{M}_{0,2 g+n} / G$. Thus (0.3) implies the following result:

(0.5) Corollary. $D \in \operatorname{Pic}\left(\bar{M}_{g, n}\right)$ is nef iff $\left.D\right|_{\bar{R}_{g, n} \cup E}$ is nef. Equivalently, the natural map

$$
\overline{N E}_{1}\left(\bar{M}_{0,2 g+n} / G \cup E\right)=\overline{N E}_{1}\left(\bar{M}_{0,2 g+n} / G\right) \times \mathbb{R}^{\geq 0} \rightarrow \overline{N E}_{1}\left(\bar{M}_{g, n}\right)
$$

is surjective.

In fact we prove the following strengthening of $(0.3)$ :

(0.6) Theorem. Let $g \geq 1$ and let $N \subset \overline{N E}_{1}\left(\bar{M}_{g, n}\right)$ be the subcone generated by the strata (1)-(5) of (2.2) for $g \geq 3$, by (1) and (3)-(5) for $g=2$, and by (1) and (5) for $g=1$. Then $N$ is the subcone generated by curves $C \subset \bar{M}_{g, n}$ whose associated family of curves has no moving smooth rational components. Hence,

$$
N E_{1}\left(\bar{M}_{g, n}\right)=N+\overline{N E}_{1}\left(\bar{M}_{0, g+n} / S_{g}\right) .
$$

(See (1.2) for the meaning of moving component.) An analogous result for families whose general member has at most one singularity is given in Moriwaki00 A]. 
Of course Theorem (0.6) implies (the second formulation of) Conjecture (0.2) for any family $C \subset \bar{M}_{g, n}$ with no moving smooth rational component. In fact, $F_{1}\left(\bar{M}_{0,7}\right)$ holds, and so Conjecture (0.2) holds as long as there is no moving smooth rational component containing at least 8 distinguished (i.e. marked or singular) points.

Theorem (0.3) also has a converse which we state as follows.

(0.7) Theorem. Every nef line bundle on $\bar{M}_{0, g+n} / S_{g}$ is the pullback of a nef line bundle on $\bar{M}_{g, n}$ and $F_{1}\left(\bar{M}_{0, g+n} / S_{g}\right)$ is equivalent to $F_{1}\left(\bar{M}_{g, n}\right)$. In particular, $F_{1}\left(\bar{M}_{g}\right)$ is equivalent to $F_{1}\left(\bar{M}_{0, g} / S_{g}\right)$ and $F_{1}\left(\bar{M}_{1, n}\right)$ is equivalent to $F_{1}\left(\bar{M}_{0, n+1}\right)$.

(0.8) Theorem. The Mori cone of $\bar{F}_{g, n}$ is a face of the Mori cone of $\bar{M}_{g, n}$ : there is a nef divisor $D$ such that

$$
D^{\perp} \cap \overline{N E}_{1}\left(\bar{M}_{g, n}\right)=\overline{N E}_{1}\left(\bar{M}_{0, g+n} / S_{g}\right) .
$$

Remark. An interesting problem is to determine whether or not $F_{1}\left(\bar{M}_{g}\right)$ for $g \geq 1$ implies $F_{1}\left(\bar{M}_{0, n}\right)$ for all $n \geq 3$. For example, if one maps $\bar{M}_{0, n}$ into $\bar{M}_{g}$ by gluing one pointed curve of very different genera, then the pullback on the Picard groups will be surjective. However, it is not clear that this will be true for the nef cones.

We note that in a number of respects using $\bar{F}_{g, n}$ to reduce the general conjecture is more desirable than using $\bar{R}_{g, n}$. Analogues of Theorems (0.7) and (0.8) fail for $\bar{R}_{g, n}$, indicating that $\bar{F}_{g, n}$ is the more natural locus from the Mori theoretic point of view (e.g. one is led to consider $\bar{F}_{g, n}$ by purely combinatorial consideration of the Mori cone). Further the birational geometry of $\bar{F}_{g, n}$ is much simpler than that of $\bar{R}_{g, n}$. For example, when $n=0$, the Picard group of $\bar{M}_{0, g} / S_{g}$ has rank roughly $g / 2$ and is freely generated by the boundary divisors, while Pic $\left(\bar{M}_{0,2 g} / G\right)$ has rank roughly $g^{2} / 2$ and there is a relation among the boundary classes. Of greater geometric significance is the contrast between the cones of effective divisors: $\overline{N E}^{1}\left(\bar{M}_{0, g} / S_{g}\right)$ is simplicial and is generated by the boundary divisors (see KeelMcKernan96]), while the structure of $\overline{N E}^{1}\left(\bar{M}_{0,2 g} / G\right)$ is unclear and it is definitely not generated by boundary divisors (a counter-example is noted below).

It is straightforward to enumerate the possibilities for the one dimensional strata, and then express (0.2) as a conjectural description of the ample cone as an intersection of explicit half spaces. This description is given for $n=0$ in [Faber96 and for $g=0$ in KeelMcKernan96. We treat the general case in $\S 2$.

A fundamental problem in birational geometry is to study morphisms of a given variety to other varieties. In the projective category any such morphism is given by a semi-ample divisor - i.e. a divisor such that the linear system of some positive multiple is basepoint free. A semi-ample divisor is necessarily nef. This implication is one of the main reasons for considering nef divisors. Correspondingly, one of the main reasons that $(0.1)$ is interesting is its connection to:

Question. What are all the contractions (i.e. morphisms with geometrically connected fibers to projective varieties) of $\bar{M}_{g, n}$ ?

We have a number of results in this direction. Recall a definition from [Keel99]: For a nef divisor $D$ on a variety $X$ a subvariety $Z \subset X$ is called $D$-exceptional if the $D$-degree of $Z$ is zero, or equivalently, $\left.D\right|_{Z}$ is not big. The exceptional locus of $D$, denoted $\mathbb{E}(D)$, is the union of the exceptional subvarieties. If $D$ is semi-ample and big, this is the exceptional locus for the associated birational map. 
(0.9) Theorem. Let $D \in \operatorname{Pic}\left(\bar{M}_{g, n}\right)$ be a nef divisor, $g \geq 2$ (resp. $\left.g=1\right)$. Either $D$ is the pullback of a nef divisor on $\bar{M}_{g, n-1}$ via one of the tautological projections (resp. is the tensor product of pullbacks of nef divisors on $\bar{M}_{1, S}$ and $\bar{M}_{1, S^{c}}$ via the tautological projection for some subset $S \subset N)$ or $D$ is big and $\mathbb{E}(D) \subset \partial \bar{M}_{g, n}$.

(0.10) Corollary. For $g \geq 2$ any fibration of $\bar{M}_{g, n}$ (to a projective variety) factors through a projection to some $\bar{M}_{g, i}(i<n)$, while $\bar{M}_{g}$ has no non-trivial fibrations.

The above corollary and its proof are part of the first author's Ph.D. thesis Gibney00, written under the direction of the second author.

(0.11) Corollary. $g \geq 1$. Let $f: \bar{M}_{g, n} \rightarrow X$ be a birational morphism to a projective variety. Then the exceptional locus of $f$ is contained in $\partial \bar{M}_{g, n}$. In particular $X$ is again a compactification of $M_{g, n}$.

Note that our results give some support to the conjecture that the nef cone of $\bar{M}_{g, n}$ behaves as if it were described by Mori's cone theorem (i.e. like the cone of a $\log$ Fano variety) with dual cone generated by finitely many rational curves and having every face contractible. This is surprising, since for example $\bar{M}_{g, n}$ is usually (e.g. for $g \geq 24$ ) of general type, and conjecturally (by Lang, see CHM97]) $n o$ rational curve meets the interior of $\bar{M}_{g, n}$ for $g \geq 2$ and $n$ sufficiently big. Of course, our main results reduce Conjecture (0.2) to genus 0 , where from initial Mori theoretic considerations it is much more plausible.

We note that prior to this work very few nef (but not ample) line bundles on $\bar{M}_{g, n}$ were known. Myriad examples can be constructed using (0.3) as we indicate in $\S 6$.

The above results indicate that (regular) contractions give only a narrow view of the geometry of $\bar{M}_{g, n}$. For example, (0.10) gives that the only fibrations are (induced from) the tautological ones and, by (0.11), birational morphisms only affect the boundary. On the other hand the birational geometry should be very rich. For example, as is seen in (6.4), all but one of the elementary (i.e. relative Picard number one) extremal contractions of $\bar{M}_{g}$ are small (i.e. isomorphisms in codimension one), so there should be a wealth of flips. Also one expects in many cases to find interesting rational fibrations. For example, when $g+1$ has more than one factorization, the Brill-Noether divisor should give a positive dimensional linear system on $\bar{M}_{g}$ which is conjecturally (see [HarrisMorrison98, 6.63]) not big.

One consequence of $(0.9)$ and the fact that $\mathbb{E}(\lambda)=\partial \bar{M}_{g}$ is that $\partial \bar{M}_{g}$ is intrinsic.

(0.12) Corollary. Any automorphism of $\bar{M}_{g}$ must preserve the boundary.

In view of our results, $F_{1}\left(\bar{M}_{0, n}\right)$ is obviously of compelling interest. It is natural to wonder:

(0.13) Question. If a divisor on $\bar{M}_{0, n}$ has non-negative intersection with all one dimensional strata, does it follow that the divisor is linearly equivalent to an effective combination of boundary divisors?

A positive answer to (0.13) would imply (0.2). The analogous property does hold on $\bar{M}_{1, n}$ (see (3.3)), but the question in that case is vastly simpler as the boundary divisors are linearly independent.

It is not true that every effective divisor on $\bar{M}_{0, n}$ is an effective sum of boundary divisors. In other words, $F^{1}\left(\bar{M}_{0, n}\right)$ is false. In particular, the second author 
has shown (in support of the slope conjecture, HarrisMorrison98, 6.63]) that the pullback of the Brill-Noether divisor from $\bar{M}_{g}$ to $\bar{M}_{0,2 g}$ is a counter-example to $F^{1}\left(\bar{M}_{0,2 g+n} / G\right)$ for any $g \geq 3$ with $g+1$ composite. In fact, he has shown that for $g=3$ the unique non-boundary component of this pullback gives a new vertex of $\overline{N E}^{1}\left(\bar{M}_{0,6} / G\right)$.

Question (0.13) can be formulated as an elementary combinatorial question of whether one explicit polyhedral cone is contained in another. As this restatement might be of interest to someone without knowledge of $\bar{M}_{g}$, we give this formulation:

(0.14) Combinatorial Formulation of (0.13). Let $\mathbb{V}$ be the $\mathbb{Q}$-vector space that is spanned by symbols $\delta_{T}$ for each subset $T \subset\{1,2, \ldots, n\}$ subject to the relations

(1) $\delta_{T}=\delta_{T^{c}}$ for all $T$;

(2) $\delta_{T}=0$ for $|T| \leq 1$; and

(3) for each 4-element subset $\{i, j, k, l\} \subset\{1,2, \ldots, n\}$

$$
\sum_{i, j \in T, k, l \in T^{c}} \delta_{T}=\sum_{i, k \in T, j, l \in T^{c}} \delta_{T} .
$$

Let $N \subset \mathbb{V}$ be the set of elements $\sum b_{T} \delta_{T}$ satisfying

$$
b_{I \cup J}+b_{I \cup K}+b_{I \cup L} \geq b_{I}+b_{J}+b_{K}+b_{L},
$$

for each partition of $\{1,2, \ldots, n\}$ into 4 disjoint subsets $I, J, K, L$. Let $E \subset \mathbb{V}$ be the affine hull of the $\delta_{T}$.

Question: Is $N \subset E$ ?

In theory (0.14) can be checked, for a given $n$, by computer. For $n \leq 6$ we have done so, with considerable help from Maroung Zou, using the program Porta. Unfortunately the computational complexity is enormous, and beyond our machine's capabilities already for $n=7$. These cases $(n \leq 6)$ were proved previously (by hand) by Faber.

The remainder of the paper is organized as follows. In $\S 1$, we fix notation for various boundary divisors and gluing maps. In $\S 2$, we give a small set of generators for the cone spanned by one dimensional strata (2.2) and inequalities which cut out the dual cone of divisors (2.1) — we term each the Faber cone. The main result of $\S 3$ is (3.1) stating that a class nef on the boundary of $\bar{M}_{g, n}$ is nef. In $\S 4$ the results from the previous section are used to deduce (0.6) and hence (0.3). We also study the nefness and exceptional loci of certain divisors in order to deduce $(0.7)$ and (0.8). The proof of $(0.9)$ is contained in $\S 5$ following preparatory lemmas $((5.1)-(5.3))$ dealing with divisors on $C^{n} / G$ where $C$ is a smooth curve with automorphism group $G$. In $\S 6$, we collect some more ad hoc results. In particular, we answer a question posed by Faber (6.2) and recover the classical ampleness result of Cornalba-Harris (6.3). Finally, $\S 7$ contains a geometric reformulation of $F_{1}\left(\bar{M}_{0, n}\right)$ and a review of the evidence (to our minds considerable) for (0.2).

\section{$\S 1$. Notation}

For the most part we use standard notation for divisors, line bundles, and loci on $\bar{M}_{g, n}$. See e.g. [Faber96] and [Faber97] with the following possible exception. By $\omega_{i}$ on $\bar{M}_{g, n}, g \geq 2$, we mean the pullback of the relative dualizing sheaf of the universal curve over $\bar{M}_{g}$ by the projection given by dropping all but the $i^{\text {th }}$ point. 
Note this is not (for $n \geq 3$ ) the relative dualizing sheaf for the map to $\bar{M}_{g, n-1}$ given by dropping the $i^{t h}$ point (the symbol is used variously in the literature).

We note that the $\mathbb{Q}$-Picard group of $\bar{M}_{g, n}$ is the same in all characteristics, by Moriwaki01.

We let $N:=\{1,2, \ldots, n\}$. The cardinality of a finite set $S$ is denoted $|S|$.

For a divisor $D$ on a variety $X$ and for $Y$ a closed subset of $X$, we say that $D$ is nef outside $Y$ if $D \cdot C \geq 0$ for all irreducible curves $C \not \subset Y$.

To obtain a symmetric description of boundary divisors on $\bar{M}_{0, n}$, we use partitions $\mathcal{T}=\left[T, T^{c}\right]$ of $N$ into disjoint subsets, each with at least two elements, rather than subsets $T$. We write the corresponding boundary divisor as $\delta_{0, \mathcal{T}}$, or $\delta_{\mathcal{T}}$. For a partition $Q$ of a subset $S \subset N$ we write $\mathcal{T}>Q$ provided the equivalence relation induced by $Q$ on $S$ refines that obtained by restricting $\mathcal{T}$ to $S$. We write $Q=\left.\mathcal{T}\right|_{S}$ provided these two equivalence relations are the same.

We write $B_{k}$ for the sum of boundary divisors on $\bar{M}_{0, n} / S_{n}$ that are the image of any $\delta_{\mathcal{T}}$ with $|T|=k$ (thus $B_{k}=B_{n-k}$ ). We will abuse notation by using the same expression for its inverse image (with reduced structure) on $\bar{M}_{0, n}, \sum_{\mathcal{T},|T|=k} \delta_{\mathcal{T}}$.

We make repeated use of the standard product decomposition for strata as a finite image of products of various $\bar{M}_{g, n}$. For precise details see [Keel99, pg. 274] and [Faber97]. To describe this decomposition for boundary divisors we use the notations

$$
\tilde{\Delta}_{i, S}:=\bar{M}_{i, S \cup *} \times \bar{M}_{g-i, S^{c} \cup *} \rightarrow \Delta_{i, S}
$$

and

$$
\tilde{\Delta}_{\text {irr }}:=\bar{M}_{g-1, N \cup p \cup q} \rightarrow \Delta_{\text {irr }} .
$$

We refer to $\bar{M}_{i, S \cup *}$ (and any analogous term for a higher codimension stratum) as a factor of the stratum.

The key fact about these maps used here is the

(1.1) Lemma. The pullback to $\tilde{\Delta}_{i, S}$ of any line bundle is numerically equivalent to a tensor product of unique line bundles from the two factors. The given line bundle is nef on $\Delta_{i, S}$ iff each of the line bundles on the factors is nef. Dually, let $C$ be any curve on the product, and let $C_{i}, C_{g-i}$ be its images on the two factors (with multiplicity for the pushforward of cycles) which we also view as curves in $\bar{M}_{g, n}$ by the usual device of gluing on a fixed curve. Then, $C$ and $C_{i}+C_{g-i}$ are numerically equivalent.

Proof. The initial statement implies the other statements, and follows from the explicit formulae of Faber97.

Any curve $E$ in $\bar{M}_{g, n}$ induces a decomposition of the curves it parameterizes into a subcurve fixed in the family $E$ and a moving subcurve. Arguing inductively, (1.1) yields

(1.2) Corollary. Every curve in $\bar{M}_{g, n}$ is numerically equivalent to an effective combination of curves whose moving subcurves are all generically irreducible.

\section{$\S 2$. The FABer cone}

In this section we consider the subcone of $\overline{N E}_{1}\left(\bar{M}_{g, n}\right)$ generated by one dimensional strata, and its dual. We refer to both as the Faber cone, appending of curves, or of divisors if confusion is possible. We will call a divisor F-nef if it lies 
in the Faber cone. Of course (0.2) is then the statement that $F$-nef implies nef, or equivalently, the Mori and Faber cones of $\bar{M}_{g, n}$ are the same.

The next result describes the Faber cone of divisors as an intersection of half spaces.

In order to give a symmetric description of the cone we write $\delta_{0,\{i\}}$ for $-\psi_{i}$ in $\operatorname{Pic}\left(\bar{M}_{g, n}\right)$ (so $\delta_{0 . I}$ is defined whenever $|I| \geq 1$ ).

(2.1) Theorem. Consider the divisor

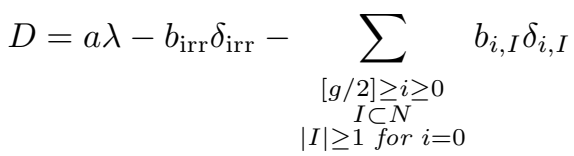

on $\bar{M}_{g, n}$ (with the convention that we omit for a given $g, n$ any terms for which the corresponding boundary divisor does not exist). Consider the inequalities

(1) $a-12 b_{\text {irr }}+b_{1, \emptyset} \geq 0$,

(2) $b_{\text {irr }} \geq 0$

(3) $b_{i, I} \geq 0$ for $g-2 \geq i \geq 0$,

(4) $2 b_{\text {irr }} \geq b_{i, I}$ for $g-1 \geq i \geq 1$,

(5) $b_{i, I}+b_{j, J} \geq b_{i+j, I \cup J}$ for $i, j \geq 0, i+j \leq g-1, I \cap J=\emptyset$,

(6) $b_{i, I}+b_{j, J}+b_{k, K}+b_{l, L} \geq b_{i+j, I \cup J}+b_{i+k, I \cup K}+b_{i+l, I \cup L}$ for $i, j, k, l \geq 0$, $i+j+k+l=g, I, J, K, L$ a partition of $N$,

where $b_{i, I}$ is defined to be $b_{g-i, I^{c}}$ for $i>[g / 2]$.

For $g \geq 3, D$ has non-negative intersection with all 1-dimensional strata iff each of the above inequalities holds.

For $g=2, D$ has non-negative intersection will all 1-dimensional strata iff (1) and (3)-(6) hold.

For $g=1, D$ has non-negative intersection with all 1-dimensional strata iff (1) and (5)-(6) hold.

For $g=0, D$ has non-negative intersection with all 1-dimensional strata iff (6) holds.

Proof. (2.2) below lists the numerical possibilities for a stratum. Each inequality above comes from standard intersection formulae (see e.g. [Faber96, [Faber97]), by intersecting with the corresponding curve of (2.2). Intersecting with (2.2.4) gives the inequality $2 b_{\text {irr }} \geq b_{i+1, I}$, which by shifting indices and notation gives (2.1.4).

Theorem (2.2) gives a listing of numerical possibilities for one dimensional strata, giving explicit representatives for each numerical equivalence class. The parts in Theorems (2.1) and (2.2) correspond: that is, for each family $X$ listed in parts (2) to (6) of (2.2), the inequality which expresses the condition that a divisor $D$ given as in (2.1) meet $X$ non-negatively is given in the corresponding part of (2.1).

We obtain these just as in [Faber96], by defining a map $\bar{M}_{0,4} \rightarrow \bar{M}_{g, n}$ by attaching a fixed pointed curve in some prescribed way. For a subset $I \subset N$, by a $k+I$ pointed curve we mean a $k+|I|$ pointed curve, where $|I|$ of the points are labeled by the elements of $I$.

(2.2) Theorem. Let $X \subset \bar{M}_{g, n}$ be a one dimensional stratum. Then $X$ is either

(1) For $g \geq 1$, a family of elliptic tails; or, numerically equivalent to the image of $\bar{M}_{0,4} \rightarrow \bar{M}_{g, n}$ defined by one of the attaching procedures (2)-(6) below.

(2) For $g \geq 3$. Attach a fixed $4+N$ pointed curve of genus $g-3$. 
(3) For $g \geq 2, I \subset N, g-2 \geq i \geq 0,|I|+i>0$. Attach a fixed $1+I$-pointed curve of genus $i$ and a fixed $3+I^{c}$-pointed curve of genus $g-2-i$.

(4) For $g \geq 2, I \subset N, g-2 \geq i \geq 0$. Attach a fixed $2+I$-pointed curve of genus $i$ and a fixed $2+I^{c}$-pointed curve of genus $g-2-i$.

(5) For $g \geq 1, I \cap J=\emptyset, I, J \subset N, i+j \leq g-1, i, j \geq 0,|I|+i,|J|+j>0$. Attach a fixed $1+I$-pointed curve of genus $i$, a fixed $1+J$-pointed curve of genus $j$, and a fixed $2+(I \cup J)^{c}$-pointed curve of genus $g-1-i-j$.

(6) For $g \geq 0,[I, J, K, L]$ a partition of $N$ into disjoint subsets, $i, j, k, l \geq 0$, $i+j+k+l=g$, and $i+|I|, j+|J|, k+|K|, l+|L|>0$. Attach $1+I, 1+J, 1+K$, and $1+L$ pointed curves of genus $i, j, k, l$ respectively.

Figure (2.3) shows schematic sketches of each of these 5 families numbered as in (2.2). The generic fiber is shown on the left of each sketch and the 3 special fibers (with, up to dual graph isomorphism, any multiplicities) are shown on the right. The bolder curves are the component(s) of the $\bar{M}_{0,4}$ piece. Boxes give the type (i.e. genus and marked point set) of each fixed component and of each node not of irreducible type.

In any of the families, if the curve sketched is not stable, we take the stabilization. Thus e.g. in (6) for $n=4, g=0$, the map $\bar{M}_{0,4} \rightarrow \bar{M}_{0,4}$ is the identity, while in (3) for $g=2, n=0, i=j=0$, the image of a generic point of $\bar{M}_{0,4}$ is the moduli point of an irreducible rational curve with two ordinary nodes.

(2)

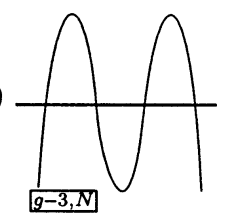

(3)

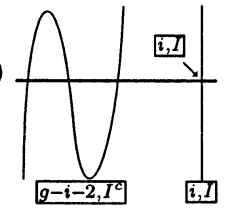

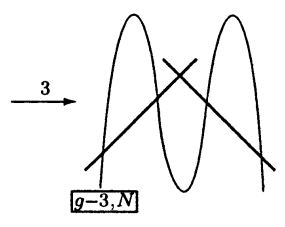
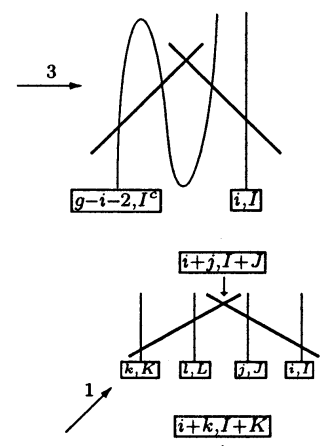

(4)
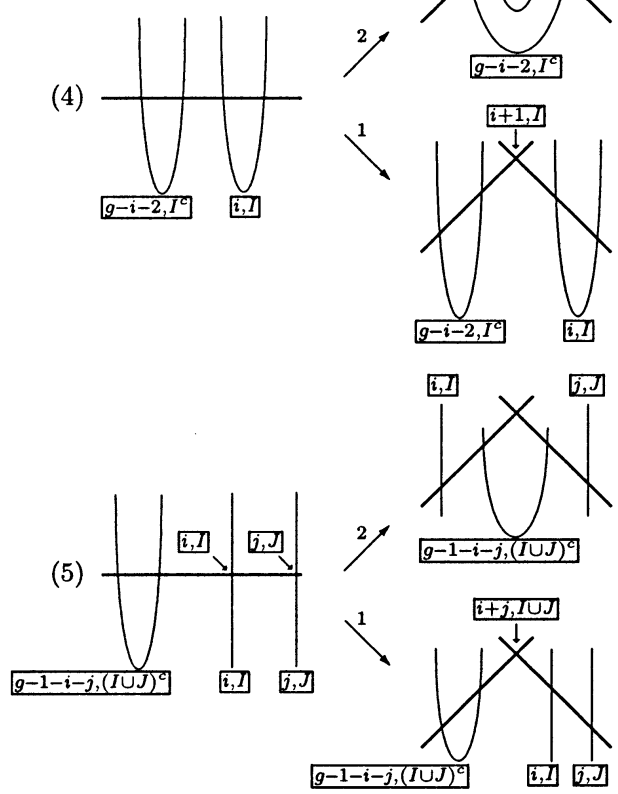

Figure (2.3). 
Strata of type (6) play distinctly different roles, both geometrically and combinatorially, from those of type (1)-(5). Those of type (6) come from the flag locus, they are the only strata in genus 0 , and there is a face of $\overline{N E}_{1}\left(\bar{M}_{0, n}\right)$ that contains exactly these strata; see (4.9). The cone generated by the strata (1)-(5) has a nice geometric meaning given in (0.6). Our proofs make no direct use of strata of type (6); in particular we will only directly use the inequalities (2.1.1)-(2.1.5).

Strata of type (2)-(6) are all (numerically equivalent to) curves lying in $\bar{R}_{g, n}$. Those of type (6) are distinguished geometrically by the fact that the curve corresponding to a general point has only disconnecting nodes, and algebraically by the fact that the corresponding inequality in (2.1) has more than 3 (in fact 7) terms.

Proof of (2.2). The proof is analogous to that of the case of $n=0$ treated in Faber96. Here are details.

Let $X \subset \bar{M}_{g, n}$ be a one dimensional stratum: $X$ is a finite image of a product of moduli spaces (one for each irreducible component of the curve $C$ corresponding to its general point). All but one of these spaces are zero dimensional, thus $\bar{M}_{0,3}$, and there is a distinguished one dimensional factor which is either $\bar{M}_{1,1}$ or $\bar{M}_{0,4}$. In the $\bar{M}_{1,1}$ case, $X$ is a family of elliptic tails and gives (2.1.1) exactly as in [Faber96].

Consider the $\bar{M}_{0,4}$ case. Let $C$ be the stable pointed curve corresponding to a general point of $X$ and let $E \subset C$ be the moving irreducible component (which is rational). Let $h: \tilde{E} \rightarrow E$ be the normalization and let $M \subset \tilde{E}$ be the union of the following disjoint subsets: $h^{-1}(Z \cap E)$ for each connected component $Z$ of the closure of $C \backslash E, h^{-1}(p)$ for each singular point $p$ of $E$, and $h^{-1}\left(p_{i}\right)$ for each labeled point $p_{i}$ of $C$ on $E$. These subsets partition $M$ into equivalence classes.

We assign to each equivalence class $\mathcal{Z}$ a triple $(a(\mathcal{Z}), S(\mathcal{Z}), h(\mathcal{Z}))$ : if $\mathcal{Z}=$ $h^{-1}(Z \cap E)$, then $h$ is the arithmetic genus of $Z, S \subset\{1,2, \ldots, n\}$ is the collection of labeled points of $C$ lying on $Z$, and $a$ is the cardinality of $Z \cap E \cdot t\left(h^{-1}(p)\right)=(2, \emptyset, 0)$ for a singular point $p$ of $E$, and $t\left(h^{-1}\left(p_{i}\right)\right)=(1,\{i\}, 0)$ for a labeled point $p_{i}$ of $C$ on $E$. The numerical class of $X$ is determined by the collection of triples $\mathcal{Z}$ by the standard intersection product formulae in, for example, Faber97. Note that $\sum_{\mathcal{Z}}(h(\mathcal{Z})+a(\mathcal{Z})-1)=g$, that $\bigcup_{\mathcal{Z}} S(\mathcal{Z})$ is a partition of $\{1,2, \ldots, n\}$, and that $\sum_{\mathcal{Z}} a(Z)=4$.

Now (2)-(6) are obtained by enumerating the possibilities for the collection of $a(Z)$ which are $\{4\},\{3,1\},\{2,2\},\{2,1,1\}$ and $\{1,1,1,1\}$. These correspond to (the obvious generalizations to pointed curves of) the families (B), (C), (D), (E), and $(\mathrm{F})$ of [Faber96] and yield (2)-(6) by considering the possibilities for $h(\mathcal{Z})$ and $S(\mathcal{Z})$ subject to the above constraints.

Throughout the paper we will separate the $\psi$ and $\delta$ classes (because although they have similar combinatorial properties, they are very different geometrically, e.g. $\psi_{i}$ is nef, while the boundary divisors have very negative normal bundles). Thus, we will write a divisor as

$$
\sum_{i=1}^{n} c_{i} \psi_{i}+a \lambda-b_{\mathrm{irr}} \delta_{\mathrm{irr}}-\sum_{\substack{S \subset N \\ n-2 \geq|S| \geq 2}} b_{0, S} \delta_{0, S}-\sum_{[g / 2] \geq j \geq 1, S \subset N} b_{i, S} \delta_{i, S}
$$

where $b_{0,\{i\}}$ has become $c_{i}$. As examples, in this notation (2.1.5) with $i=j=$ $0, I=\{i\}$ becomes

$$
c_{i}+b_{0, J} \geq b_{0, J \cup\{i\}}
$$


which in turn specializes to

$$
c_{i}+c_{j} \geq b_{0,\{i, j\}}
$$

in case $J=\{j\}$ while $(2.1 .3)$ for $(i, I)=(0,\{i\})$ becomes $c_{i} \geq 0$.

For $g \geq 3$, the expression above is unique (i.e. the various tautological classes are linearly independent), but for smaller genera there are relations which are listed in ArbarelloCornalba98. For $g=1$, the boundary divisors are linearly independent and span the Picard group so we can assume above that $a$ and all $c_{i}$ are zero and, if we do so, the resulting expression is unique.

\section{§3. NEF ON THE BOUNDARY IMPLIES NEF}

In this section, we prove:

(3.1) Proposition. If $g \geq 2$, or $g=1, n \geq 2$, a divisor $D \in \operatorname{Pic}\left(\bar{M}_{g, n}\right)$ is nef iff its restriction to $\partial \bar{M}_{g, n}$ is nef.

(3.2) Lemma. $g \geq 2$. Let $D \in \operatorname{Pic}\left(\bar{M}_{g, n}\right)$ be a divisor expressed as in (2.1). For $[g / 2] \geq i \geq 1$ let

$$
b_{i}=\max _{S \subset N} b_{i, S}
$$

and define $A \in \operatorname{Pic}\left(\bar{M}_{g}\right)$ by

$$
A=a \lambda-b_{\text {irr }} \delta_{\text {irr }}-\sum_{[g / 2] \geq i \geq 1} b_{i} \delta_{i} .
$$

If the coefficients of D satisfy one of the inequalities (2.1.1)-(2.1.5) for fixed indices $i, j$ and all subsets of $N$, then the coefficients of $A$ satisfy the analogous inequality. In the example of (2.1.5), $b_{i}+b_{j} \geq b_{i+j}$ if $b_{i, I}+b_{j, J} \geq b_{i+j, I \cup J}$ for all $i$ and $j$ between 0 and $g-1$ and for all subsets $I, J \subset N$ with $I \cap J=\emptyset$.

Proof. This is clear since the $b_{i}$ are defined by taking a maximum. For example, suppose $b_{i+j}=b_{i+j, T}$. Then, $b_{i}+b_{j} \geq b_{i, \emptyset}+b_{j, T} \geq b_{i+j, T}=b_{i+j}$.

Since all the strata are contained in $\partial \bar{M}_{g, n},(3.1)$ will follow immediately from:

(3.3) Lemma. Let $D \in \operatorname{Pic}\left(\bar{M}_{g, n}\right)$. If $g \geq 3[$ resp. $g=2 ; g=1]$ and $D$ has non-negative intersection with all strata in (2.2) of types (1)-(5) [resp. (1)-(2) and (4)-(5); (1) and (5)], then $D$ is nef outside of $\partial \bar{M}_{g, n}$. Furthermore if $g=1$, then $D$ is numerically equivalent to an effective sum of boundary divisors.

Proof. We express $D$ as in (2.4). We consider first the case of $g=1$. We can as remarked above assume $a=c_{i}=0$ for all $i$. Then it is immediate from (2.1.1) and (2.1.5) (using the translations after (2.4)) that all the coefficients are non-positive, so $D$ is linearly equivalent to an effective sum of boundary divisors.

Now assume $g \geq 2$. Define $b_{i}$ and $A$ as in (3.2).

We use the relation

$$
\psi_{i}=\omega_{i}+\sum_{i \in S} \delta_{0, S}
$$


(which, as in [ArbarelloCornalba96], is obtained easily from the definition by induction on $n$ ) to write

$$
D=\sum_{i \in N} c_{i} \omega_{i}+\left(\sum_{\substack{S \subset N \\ n-2 \geq|S| \geq 2}}\left(\left(\sum_{i \in S} c_{i}\right)-b_{0, S}\right) \delta_{0, S}\right)+\pi^{*}(A)+E
$$

where $\pi$ is the forgetful map to $\bar{M}_{g}$ and $E$ is a combination of boundary divisors $b_{i, S}, i>0-$ an effective one since we take the maximum in forming $b_{i}$.

By repeated application of (2.1.5) as translated below (2.4), the coefficients of the $\delta_{0, S}$ in (3.4) are non-negative. By (2.1.3), $c_{i} \geq 0$. Since each $\omega_{i}$ is nef - by, e.g. [Keel99] - it's enough to show $A$ is nef outside $\partial \bar{M}_{g}$.

Suppose first $g \geq 3$. Then $A$ satisfies each of (2.1.1)-(2.1.5) by (3.2). Thus $a \geq 10 b_{\text {irr }} \geq 0$ and $a \geq 5 b_{i}$, so the claim follows from (3.5) below. Now suppose $g=2$. By (3.2) and (2.2) $a-10 b_{\text {irr }} \geq 2 b_{\text {irr }}-b_{1} \geq 0$ so the claim follows from [Faber96, §2].

(3.5) Lemma. Let $D=a \lambda-\sum b_{i} \delta_{i}$ be a divisor on $\bar{M}_{g}$, and let $g: Z \rightarrow \bar{M}_{g}$ be a morphism from a projective variety such that $g(Z) \not \subset \partial \bar{M}_{g}$. Assume $g \geq 2$. Consider the inequalities:

(1) $a \geq 0$.

(2) $g \cdot a \geq(8 g+4) b_{\text {irr }}$.

(3) $2 g \cdot a \geq(8 g+4) b_{i},[g / 2] \geq i \geq 1$.

If (1)-(3) hold, then $g^{*}(Z)$ is a limit of effective Weil divisors, and if strict inequality holds in each case and $g$ is generically finite, then $g^{*}(Z)$ is big.

Proof. The final statement follows from the previous statements using $12 \lambda=\kappa+\delta$ and the ampleness of $\kappa$.

Let $\Gamma$ be the divisor of CornalbaHarris88, 4.4]

$$
(8 g+4) \lambda-g \delta_{\text {irr }}-2 g \sum_{i>0} \delta_{i} .
$$

The inequalities imply $D=s \lambda+c \Gamma+E$ where $c, s \geq 0$ and $E$ is an effective sum of boundary divisors. The pullback $g^{*}(\Gamma)$ is a limit of effective Weil divisors by a slight generalization of [CornalbaHarris88, 4.4] (in characteristic zero we could apply Moriwaki98 directly). When $g(Z)$ is contained in the hyperelliptic locus, their analysis applies without change, as long as the characteristic is not two. When $g(Z)$ is not contained in the hyperelliptic locus, one needs the generalization of their (2.9) to smooth $T$ of arbitrary dimension (with their $e_{L}(\mathcal{I})$ replaced by the analogous expression supported at codimension one points of $T$ and the conclusion being that the expression is a limit of effective Weil divisors, as in their (1.1)). Their proof extends after obvious modifications.

(3.6) Theorem. Let $D \in \operatorname{Pic}\left(\bar{M}_{g, n}\right)$ be a divisor with non-negative intersection with all 1-strata of type (2.2.1)-(2.2.5). Let $C \subset \bar{M}_{g, n}$ be a complete one dimensional family of curves, whose generic member is a curve without moving rational components. Then $D \cdot C \geq 0$. If furthermore $\left.D\right|_{\bar{R}_{g, n}}$ is nef, then $D$ is nef.

Proof. We induce simultaneously on $g, n$. For $g=0$ or $g=1, n=1$ there is nothing to prove. So suppose $g \geq 2$, or $g=1, n \geq 2$.

Suppose first that $D$ is nef on all 1-strata of type (1)-(5). Let $C$ be as in the statement. We will show that $D \cdot C \geq 0$. By (3.3) we may assume $C \subset \partial \bar{M}_{g, n}$. 
Suppose $C \subset \Delta_{i, S}$ for $i>0$. We apply induction using (1.1), following that notation. It's enough to show $D_{i} \cdot C_{i} \geq 0$. A stratum of type (1)-(5) on one of the factors yields (by the usual inclusion given by gluing on a fixed curve) a stratum of type (1)-(5) on $\bar{M}_{g, n}$, so $D_{i}, C_{i}$ satisfy the induction hypothesis. If $C \subset \Delta_{\text {irr }}$ we can pass to $\tilde{\Delta}_{\text {irr }}$ and apply induction directly.

Now suppose in addition that $\left.D\right|_{\bar{R}_{g, n}}$ is nef. We use induction just as above (with the same notation). Note that we may assume that $D_{i}$ is nef on $\bar{R}_{i, S \cup *}$, since we may choose the fixed curve we glue on to be rational.

\section{$\S 4$. Reduction to the Flag Locus}

(4.1) Proposition. Let $D$ be a divisor on $\bar{M}_{g, n}, g \geq 1$, satisfying the hypothesis of (3.3). Let $X \subset \bar{R}_{g, n}$ be a stratum whose general member corresponds to a stable curve with no disconnecting nodes. Then $\left.D\right|_{X}$ is linearly equivalent to an effective sum of boundary divisors and nef divisors.

(4.2) Corollary. Let $C \subset \bar{M}_{g, n}$ be a complete one dimensional family of pointed curves whose general member has no moving smooth rational components. Let $D$ be a divisor on $\bar{M}_{g, n}$. If $D$ has non-negative intersection with all 1-strata of type (2.2.1)-(2.2.5), then $D \cdot C \geq 0$.

Proof. We use induction as in the proof of (3.6). By (1.2) we can assume that the general member of $C$ is irreducible, and by (3.6), rational. Now (4.1) applies.

\section{(4.3) Corollary.}

$$
N E_{1}\left(\bar{M}_{g, n}\right)=N+\overline{N E}_{1}\left(\bar{M}_{0, g+n} / S_{g}\right)
$$

where $N$ is the subcone generated by the strata (2.2.1)-(2.2.5).

Proof. Immediate from (4.2), (1.1) and (1.2).

Of course (4.2) and (4.3) together contain (0.6), which in turn implies (0.3).

Proof of (4.1). First we reduce to the case of $n=0$. As in (3.4) we have

$$
D=\sum_{i \in N} c_{i} \omega_{i}+\pi^{*}(A)+G
$$

with $G$ an effective combination of boundary divisors parameterizing degenerations with a disconnecting node. (In the genus 1 case the sum is empty and $A$ is a multiple of $\delta_{\text {irr }}$ ). So we can replace $X$ by its image under $\pi$. When $g=1$ this is a point and there is nothing to prove. When $g=2$ this image is either a point, or is one dimensional and defines inequality (2.1.4) (so the pullback of $D$ is nef). So we can assume $n=0, g \geq 3$.

Let $C$ be the stable curve corresponding to a general point of $X$, and let $E$ be a component of $C$. As in the proof of (2.2) we get from $E$ a map of some $\bar{M}_{0, n}$ into $\bar{M}_{g}$ and we want to see that the pullback of $D$ is an effective sum of boundary components and nef divisors. As in the proof of (2.2), the $n$ points are divided into equivalence classes: the singular points of $E$ are divided into disjoint 2-cycles, while the points where $E$ meets other irreducible components of $C$ are divided by the connected components of (the closure of) $C \backslash E$, each class with at least two elements by our assumption that $C$ has no disconnecting nodes. If $P$ is the corresponding partition, then a class $\delta_{i}, i>0$, pulls back to a sum of irreducible 
divisors $\delta_{\mathcal{T}}$ with $\mathcal{T}>P$ (i.e. $P$ refining the partition $\left[T, T^{c}\right]$ ). We use the identity on $\bar{M}_{g}$

$$
-\delta_{\mathrm{irr}}+12 \lambda=\kappa+\sum_{i>0} \delta_{i}
$$

and the fact that $\lambda$ is trivial on $\bar{M}_{0, n}$. If $b_{\text {irr }} \leq 0$, then $b_{\text {irr }}=0$ by (2.1.2) and so the result is clear from (2.1.4). So we may assume (by scaling) that $b_{\mathrm{irr}}=1$. Then $D$ pulls back to

$$
\kappa+\sum_{\mathcal{T}>P} e_{T} \delta_{\mathcal{T}}
$$

with $e_{T} \geq-1$ by (2.1.4). Now apply (4.4) below.

(4.4) Lemma. Let $P$ be a partition of $\{1,2, \ldots, n\}$ such that each equivalence class contains at least two elements. The divisor

$$
\kappa+\sum_{\mathcal{T}>P} e_{\mathcal{T}} \delta_{\mathcal{T}}
$$

on $\bar{M}_{0, n}$ is linearly equivalent to an effective sum of boundary divisors, if each $e_{\mathcal{T}} \geq-1$.

Remark. Note that at least some condition on $P$ is required. E.g. $\kappa-\delta$ is not an effective sum of boundary divisors, since as a symmetric expression the coefficient of $B_{2}$ is negative, and there are no symmetric relations.

Proof of (4.4). Expanding $\kappa$ in terms of boundary divisors (see [KeelMcKernan96]) we have

$$
\sum_{\mathcal{T}>P}\left(\frac{|T|(n-|T|)}{n-1}+e_{\mathcal{T}}-1\right) \delta_{\mathcal{T}}+\sum_{\mathcal{T} \ngtr P}\left(\frac{|T|(n-|T|)}{n-1}-1\right) \delta_{\mathcal{T}} .
$$

Note $\frac{|T|(n-|T|)}{n-1} \geq 2$ unless $|T|=2$, in which case it is $2-\frac{2}{n-1}$, or $n=6$ and $|T|=3$ in which case it is $2-\frac{1}{n-1}$. So the sum is nearly effective as it stands. We use relations in the Picard group to deal with the negative coefficients.

We assume first $n \geq 7$. If there are no 2-cycles in $P$ our sum is already effective, so (after perhaps reordering) we can write $P$ as

$$
(12)(34) \ldots(2 k-12 k)
$$

for some $k \geq 1$. Further we can assume either that $2 k=n$, or we have a further $t$-cycle, $t \geq 3,(2 k+12 k+2 \ldots 2 k+t)$. We use the $k$ relations

$$
\sum_{[\{i, i+1\},\{i+2, i+3\}]=\left.\mathcal{T}\right|_{\{i, i+1, i+2, i+3\}}} \delta_{\mathcal{T}}=\sum_{[\{i, i+2\},\{i+1, i+3\}]=\left.\mathcal{T}\right|_{\{i, i+1, i+2, i+3\}}} \delta_{\mathcal{T}}
$$

for $i=1,3,5, \ldots, 2 k-1$ with the adjustment that if $2 k=n$, then we replace the last by the analogous relation for $[\{2 k-1,2 k\},\{1,2\}]$. By $[\{i, i+1\},\{i+2, i+3\}]=$ $\left.\mathcal{T}\right|_{\{i, i+1, i+2, i+3\}}$ we mean that the restriction of $\mathcal{T}$ to the 4 -element set is the given partition into disjoint 2-cycles, i.e. either $i, i+1 \in T$ and $i+2, i+3 \in T^{c}$ or this holds with $T$ and $T^{c}$ reversed. Thus the above relations come directly from those in [Keel92] by adjusting notation.

Let $L=R$ be the relation among effective boundary divisors that one obtains by adding together the $k$ relations above (without canceling terms common to both sides). Let $\delta_{\text {irr }}$ be the sum of the $\delta_{\mathcal{T}}$ with $\mathcal{T} \ngtr P$, and let $\delta_{1}$ be the sum of those 
with $\mathcal{T}$ one of the 2-cycles of $P$ together with its complement. Note $R$ is supported on $\delta_{\text {irr }}$ while $L \geq \delta_{1}$ (here and throughout the paper inequalities between sums of Weil divisors stand for a system of inequalities, one for each component) and $L \geq 2 \delta_{1}$ when $n=2 k$.

Choose $s>0$ maximal so that

$$
s R \leq \sum_{\mathcal{T} \ngtr P}\left(\frac{|T|(n-|T|)}{n-1}-1\right) \delta_{\mathcal{T}} .
$$

Thus (4.5) is linearly equivalent to

$$
s L-\frac{2}{n-1} \delta_{1}+E
$$

for $E$ an effective sum of boundary divisors.

Define coefficients $\alpha_{\mathcal{T}}$ so $R=\sum_{\mathcal{T}} \alpha_{\mathcal{T}} \delta_{\mathcal{T}}$.

Since $L \geq \delta_{1}$, it is enough to show that $s \geq \frac{2}{n-1}$, or equivalently that

$$
\frac{2}{n-1} \alpha_{\mathcal{T}} \leq \frac{|T|(n-|T|)}{n-1}-1
$$

and for $n=2 k, \frac{\alpha_{\mathcal{T}}}{n-1} \leq \frac{|T|(n-|T|)}{n-1}-1$ is sufficient. Suppose first $|T|=2$. Note that $\delta_{0, T}$ can occur (with non-zero coefficient) on the right hand side of at most one of the $k$ relations. Thus $\alpha_{\mathcal{T}} \leq 1$ and (4.6) follows.

Suppose now $|T| \geq 3$. Then (4.6) holds as long as $2 \alpha_{\mathcal{T}} \leq n-1$. Since $\alpha_{\mathcal{T}} \leq k$, we can assume $2 k=n$. Then we only need $\alpha_{\mathcal{T}} \leq 2 k-1$, which holds as $\alpha_{\mathcal{T}} \leq k$.

Now we consider the cases of $n \leq 6$. If $P$ has an $n$-cycle, the result is obvious $(\mathcal{T}>P$ is then impossible). Up to reordering, the possibilities are $(12)(34)$, $(12)(345),(12)(34)(56)$ and $(123)(456)$. In the first case (4.5) is nef (and we are working on $\left.\bar{M}_{0,4}=\mathbb{P}^{1}\right)$. In the second case the above argument applies without change. In the third we can combine the above argument for the cases $n=2 k$ and $|T|=2$. Finally consider $P=(123)(456)$. Thus $\mathcal{T}>P$ implies $\delta_{\mathcal{T}}=\delta_{0,\{1,2,3,\}}$ We use the relation

$$
\sum_{[\{1,2\},\{5,6\}]=\left.\mathcal{T}\right|_{\{1,2,5,6\}}} \delta_{\mathcal{T}}=\sum_{[\{1,5\},\{2,6\}]=\left.\mathcal{T}\right|_{\{1,2,5,6\}}} \delta_{\mathcal{T}}
$$

to show (4.5) is effective as above.

(4.7) Theorem. Consider the line bundle

$$
D=a \lambda+\sum_{i=1}^{i=n}(g+n-1) \psi_{i}-b_{\mathrm{irr}} \delta_{\mathrm{irr}}-\sum(g+n-(i+|S|))(i+|S|) \delta_{i, S}
$$

on $\bar{M}_{g, n}$.

Then, $\left.D\right|_{F_{g, n}}$ is trivial and $D$ has zero intersection with all strata of type (2.2.6). Moreover, $D$ has strictly positive intersection with all other strata if $a>12 b_{\mathrm{irr}}-$ $(g+n-1), 2 b_{\mathrm{irr}}>((n+g) / 2)^{2}$. If $D$ satisfies these inequalities, $D$ is nef. In particular $F_{1}\left(\bar{M}_{g, n}\right)$ implies $F_{1}\left(\bar{M}_{0, g+n} / S_{g}\right)$.

Every nef line bundle on $\bar{M}_{0, g+n} / S_{g}$ is the pullback of a nef line bundle on $\bar{M}_{g, n}$. The strata numerically equivalent to a curve in the flag locus are precisely the strata of type (2.2.6). 
Proof. One checks by (2.1.6) that $D$ has trivial intersection with all the strata of type (2.2.6). These correspond to the strata of $\bar{M}_{0, g+n}$. Since the strata in $\bar{M}_{0, n}$ generate the Chow group, it follows that $D$ has trivial pullback, as in $(0.3)$, to $\bar{M}_{0, g+n} / S_{g}$. Now one checks using (2.1)-(2.2) that $D$ has strictly positive intersection with all other strata if the inequalities in the statement hold. The final remark in the statement now follows.

Now suppose the inequalities of the statement hold. Then $D$ is nef by $(0.3)$. Standard intersection formulae (e.g. [Faber97]) show that

$$
f^{*}: \operatorname{Pic}\left(\bar{M}_{g, n}\right) \rightarrow \operatorname{Pic}\left(\bar{M}_{0, g+n} / S_{g}\right)
$$

is surjective. Suppose $f^{*}(W)$ is nef. Then $W+m D$ will have non-negative intersection with all strata for $m>>0$ by the above. Thus it is nef by $(0.3)$.

Of course the above theorem implies (0.7).

(4.8) Lemma. Let $D=D_{g, n}$ be the divisor of (4.7). Let $h: \bar{M}_{j, s} \rightarrow \bar{M}_{g, n}$ be a factor of one of the strata. If $h$ corresponds to a smooth rational component, then $h^{*}(D)$ is trivial. Otherwise $\mathbb{E}\left(h^{*}(D)\right) \subset \partial \bar{M}_{j, s}$.

Proof. In the first case we can assume (without affecting the pullback on Picard groups) that $h$ comes from a stratum in the flag locus, and so the result follows from (4.7).

Now assume either $j \geq 1$, or $j=0$ and the corresponding component contains at least one disconnecting node. If $s \geq 1$, let $C$ be the general fiber of one of the projections $\bar{M}_{j, s} \rightarrow \bar{M}_{j, s-1}$. Then, $C$ is numerically equivalent to an effective sum of strata, not all of which are of type (2.2.6). Thus $h^{*}(D) \cdot C>0$ by (4.7). Now (0.10) applies as long as $j \geq 2$.

Suppose $j=1$. Observe by (4.7) that $d D_{j, s}-h^{*}(D)$ is nef for sufficiently large $d$. Thus it's enough to show $\mathbb{E}\left(D_{j, s}\right) \subset \partial \bar{M}_{j, s}$. By (4.7) and the proof of (3.3), $D_{1, s}$ is an effective sum of boundary divisors, with every divisor occurring with positive coefficient. Since the boundary supports an ample divisor, the result follows.

Now assume $j=0$, and the corresponding component contains a disconnecting node. As in the previous paragraph, we can assume $h$ defines $\bar{R}_{g, n}, g \geq 1$. By (4.7), for any divisor $W, d D-W$ satisfies the hypothesis of (3.3). So, taking $W$ ample, the result follows from (4.1).

(4.9) Corollary. If $D$ is the nef divisor of (4.7), then

$$
D^{\perp} \cap \overline{N E}_{1}\left(\bar{M}_{g, n}\right)=\overline{N E}_{1}\left(\bar{M}_{0, g+n} / S_{g}\right) .
$$

Proof. Let $C \subset \bar{M}_{g, n}$ be a curve. By (4.8) and (1.2), D $C=0$ iff every moving component of the associated family of curves is smooth and rational, in which case $C$ is algebraically equivalent to a curve in $\bar{F}_{g, n}$.

\section{$\S 5$. EXCEPTIONAL LOCI}

In this section we prove (0.9). We will use the following lemmas.

(5.1) Lemma. Let $F$ be the fiber of $\pi: \bar{M}_{g, n} \rightarrow \bar{M}_{g}$ (resp. $\pi: \bar{M}_{1, n} \rightarrow \bar{M}_{1,1}$ ) over a point $[C]$ (resp. $[C, p]$ ) with $C$ smooth. Let $G$ be the automorphism group of $C$. Let $D$ be a divisor on $F$ which is the restriction of a nef divisor in $\operatorname{Pic}\left(\bar{M}_{g, n}\right)$. Let

$$
f: F \rightarrow C^{n} / G
$$


be the natural birational map. $D$ is big iff $f_{*}(D)$ is big, in which case

$$
\mathbb{E}(D) \subset f^{-1}\left(\mathbb{E}\left(f_{*}(D)\right)\right) \cup \partial F .
$$

Proof. We will abuse notation slightly and use the same symbol for a divisor on $\bar{M}_{g, n}$ and its restriction to $F$.

Suppose first $g \geq 2$. As in (3.4) we have an expression

$$
D=\sum_{i \in N} c_{i} \omega_{i}+\left(\sum_{\substack{S \subset N \\ n-2 \geq|S| \geq 2}}\left(\left(\sum_{i \in S} c_{i}\right)-b_{0, S}\right) \delta_{0, S}\right)
$$

with $c_{i} \geq 0$, and with each $\delta_{0, S}$ having non-negative coefficient. Let

$$
\Gamma=\left(\sum_{\substack{S \subset N \\ n-2 \geq|S| \geq 2}}\left(\left(\sum_{i \in S} c_{i}\right)-b_{0, S}\right) \delta_{0, S}\right) .
$$

Observe that the exceptional divisors of $f$ are precisely the $\delta_{0, S}$ with $|S| \geq 3$, and that $f\left(\delta_{0, S}\right) \subset f\left(\delta_{0, T}\right)$ iff $T \subset S$. Thus the support of $f^{-1}\left(f_{*}(\Gamma)\right)$ is the union of the $\delta_{0, S}$ over subsets $S$ which contain a 2-element subset, $T$, such that $\delta_{0, T}$ occurs in $\Gamma$ with non-zero coefficient. By repeated application of (2.1.5), we obtain

$$
\left(\sum_{i \in S} c_{i}\right)-b_{0, S} \geq\left(\sum_{i \in T} c_{i}\right)-b_{0, T}
$$

if $T \subset S$. Thus the support of $\Gamma$ contains the support of $f^{-1}\left(f_{*}(\Gamma)\right)$ (in fact since $D$ is $f$-nef, by negativity of contractions, the supports are the same). As each $\omega_{i}$ is a pullback along $f$ it follows that $D$ is big iff $f_{*}(D)$ is big. By [Keel99, 4.9], $\omega_{i}$ is nef and $\mathbb{E}\left(\omega_{i}\right) \subset \partial F$. The result follows.

Now suppose $g=1$. By (3.3) we may express $D$ as

$$
-\sum b_{0, S} \delta_{0, S}
$$

with $b_{0, S} \leq 0$. Using (2.1) one checks $b_{0, S} \leq b_{0, T}$ for $T \subset S$. Now it follows as above that $D$ has the same support as $f^{-1}\left(f_{*}(D)\right)$. The result follows.

(5.2) Lemma. Let $C$ be a smooth curve of genus $g \geq 2$, with automorphism group $G$. Let $D$ be an divisor on $C^{n} / G$ of the form

$$
D=\sum c_{i} \omega_{i}+\sum_{i \neq j} a_{i j} \Delta_{i j}
$$

with $c_{i}, a_{i j} \geq 0$ and $c_{i}+c_{j} \geq a_{i j} .\left.D\right|_{Z}$ is big for any subvariety $Z \subset C^{n} / G$ not contained in any diagonal iff $D$ is $q_{i}$-relatively ample for each of the projections

$$
q_{i}: C^{n} / G \rightarrow C^{n-1} / G
$$

given by dropping the $i^{\text {th }}$ point.

Proof. The forward implication is obvious. Consider the reverse implication. As $G$ is finite it's enough to consider the analogous statements on $C^{n}$. We assume $D$ is $q_{i}$-ample for all $i$. Write

$$
D=\sum c_{i} \omega_{i}+\sum a_{i j} \Delta_{i j}
$$


Reorder so that $c_{i}=0$ iff $i \geq s+1$. For each $t>s$, since $D$ is $\pi_{t}$-ample, $a_{i(t) t}>0$ for some $i(t) \neq t$. Since $c_{i}+c_{j} \geq a_{i j} \geq 0$, we must have $i(t) \leq s$. If

$$
p: C^{n} \rightarrow C^{s}
$$

is the projection onto the first $s$ factors, then $\sum c_{i} \omega_{i}$ is the pullback along $p$ of an ample divisor, while

$$
\sum_{t>s} a_{i(t) t} \Delta_{i(t) t}
$$

is $p$-ample.

(5.3) Lemma. Let $C$ be a smooth curve of genus 1, with automorphism group $G$. Let

$$
D=\sum a_{i j} \Delta_{i j}
$$

be an effective combination of diagonals on $C^{n} / G$. Let $H \subset S_{n}$ be the subgroup generated by the (ij) with $a_{i j} \neq 0$. If $H$ is transitive, then $D$ is ample. If a partition $N=S \cup S^{c}$ is preserved by $H$, then $D$ is pulled back along the fibration

$$
C^{n} / G \rightarrow C^{S} / G \times C^{S^{c}} / G .
$$

Proof. The second claim is clear. For the first we induce on $n$. For $n=2$ the result is clear (the quotient is a curve and $D$ is non-trivial). Note since the genus is one, that all the diagonals (and hence $D$ ) are semi-ample, so it's enough to show $D$ has positive intersection with every irreducible curve. But if $\Delta_{i j} \cdot E=0$ for a curve $E$, then $E$ is numerically equivalent to a curve contained in $\Delta_{i j}$ so it's enough to show $\left.D\right|_{\Delta_{i j}}$ is ample, and for this we can apply induction.

Proof of (0.9). Suppose first $g \geq 2$. If $n=0$ and $g=2$, the result follows from Faber96. If $n=0$ and $g \geq 3$, we apply (3.5). If $a=0$, then $D$ is trivial by (2.1.1)-(2.1.4). Otherwise we have strict inequality in (3.5) by (2.1).

So we may assume $n>0$ and that $D$ is not pulled back from $\bar{M}_{g, n-1}$. If $D$ is trivial on the general fiber of $q: \bar{M}_{g, n} \rightarrow \bar{M}_{g, n-1}$, then it is $q$-trivial, since it is nef, thus, by an easy calculation, pulled back. So we can assume $D$ is $q$-big (for any choice of $q$ ).

Mimicking the argument of (3.3) we write $D$ as in (3.4)

$$
D=\left(\sum_{i \in s} c_{i} \omega_{i}\right)+\left(\sum_{\substack{S \subset\{1,2, \ldots, n\} \\ n-2 \geq|S| \geq 2}}\left(\left(\sum_{i \in S} c_{i}\right)-b_{0, S}\right) \delta_{0, S}\right)+\pi^{*}(A)+E .
$$

For any subvariety $Z \not \subset \partial \bar{M}_{g},\left.A\right|_{Z}$ is effective by (3.5). Thus by [KollarMori98, 3.23] it is enough to show that $\mathbb{E}\left(\left.D\right|_{F}\right) \subset F \cap \partial \bar{M}_{g, n}$ for $F$ as in (5.1). This is immediate from (5.1)-(5.2).

Now suppose $g=1$. Suppose first that $D$ is $q_{S}$ big for each fibration

$$
q_{S}: \bar{M}_{1, n} \rightarrow \bar{M}_{1, S} \times \bar{M}_{1,1} \bar{M}_{1, S^{c}}
$$

and each non-trivial proper subset $S \subset N$. We conclude as in the $g \geq 2$ case by applying (5.1) and (5.3). 
So we may assume we have a non-trivial proper subset $S \subset N$ such that $D$ has zero intersection with the general fiber (which is one dimensional) of $q_{S}$. As $D$ is nef, a short calculation shows $D$ is of the form $\pi_{S}^{*}\left(D_{S}\right)+\pi_{S^{c}}^{*}\left(D_{S^{c}}\right)$ for divisors $D_{S}$ and $D_{S^{c}}$ on $\bar{M}_{1, S}$ and $\bar{M}_{1, S^{c}}$. Indeed one checks with (2.2.5) that

$$
b_{0, T \cap S}+b_{0, T \cap S^{c}}=b_{0, T}
$$

for all $T$, from which it follows that we may take

$$
\begin{aligned}
D_{S} & =-\sum_{T \subset N} b_{0, T \cap S} \delta_{0, T \cap S}, \\
D_{S^{c}} & =-\sum_{T \subset N} b_{0, T \cap S^{c}} \delta_{0, T \cap S^{c} .}
\end{aligned}
$$

Now note that $\bar{R}_{1, m}$ and all of the 1-strata of $\bar{M}_{1, n}$ except for the elliptic tails lie over the point $\delta_{\text {irr }} \in \bar{M}_{1,1}$. It follows from (3.6) that for divisors $D_{S} \in \operatorname{Pic}\left(\bar{M}_{1, S}\right)$ and $D_{S^{c}} \in \operatorname{Pic}\left(\bar{M}_{1, S^{c}}\right)$, if $\pi_{S}^{*} D_{S}+\pi_{S^{c}}^{*} D_{S^{c}}$ is nef, then both $D_{S}$ and $D_{S^{c}}$ are nef, as long as each has non-negative intersection with a family of elliptic tails. Let $E \subset \bar{M}_{1, n}$ be a family of elliptic curves. Then so are $E_{S}=\pi_{S}(E)$ and $E_{S^{c}}=\pi_{S^{c}}(E)$ and $\delta_{\text {irr }} \cdot E_{S}=\delta_{\text {irr }} \cdot E_{S^{c}}>0$. So if we define $t$ by

$$
\left(D_{S}+t \delta_{\text {irr }}\right) \cdot E_{S}=0
$$

then

$$
\left(D_{S^{c}}-t \delta_{\mathrm{irr}}\right) \cdot E_{S^{c}}=D_{S} \cdot E_{S}+D_{S^{c}} \cdot E_{S^{c}}=D \cdot E \geq 0
$$

Hence

$$
D=\pi_{S}^{*}\left(D_{S}+t \delta_{\mathrm{irr}}\right)+\pi_{S^{c}}^{*}\left(D_{S^{c}}-t \delta_{\mathrm{irr}}\right)
$$

with both $D_{S}+t \delta_{\text {irr }}$ and $D_{S^{c}}-t \delta_{\text {irr }}$ nef.

Remarks. A natural analog of $f$ from (5.1) for $g=0$ is the birational map

$$
f: \bar{M}_{0, n} \rightarrow Q
$$

with $Q$ one of the maximal GIT quotients of $\left(\mathbb{P}^{1}\right)^{\times n}$ (e.g. the unique $S_{n}$ symmetric quotient). The birational geometry of $Q$ is well understood (see [HuKeel00]), and in particular the analog of (5.3) holds. Thus if one could prove the analog of (5.1), one could extend (0.9) to all genera. This would imply (0.2).

\section{$\S 6$. AD HOC EXAMPLES}

(6.1) Proposition. (char 0) Let $D$ be an F-nef divisor $a \lambda-\sum b_{i} \delta_{i}$ on $\bar{M}_{g}$. Assume further for each coefficient $b_{i},[g / 2] \geq i \geq 1$, that either $b_{i}=0$ or $b_{i} \geq b_{\text {irr }}$. Then $D$ is nef.

Proof. By (0.3) it is enough to show $h^{*}(D)$ is nef, for the composition $h: \bar{M}_{0,2 g} \rightarrow$ $\bar{R}_{g, n} \subset \bar{M}_{g}$. We can assume $b_{\text {irr }}>0$ (otherwise all $b_{i}=0$ ). Suppose first $b_{i}=0$ for some $i>0$. Consider the map

$$
\bar{M}_{i, 1} \rightarrow \bar{M}_{g}
$$


(from the usual product decomposition of $\delta_{i}$ ). Let $D^{\prime}$ be the pullback of $D$, which we express in the usual basis. Intersecting with an appropriate stratum of type (2.1.5) on $\bar{M}_{i, 1}$ gives the inequality

$$
c+b_{j, \emptyset} \geq b_{j,\{1\}}=b_{g-j, \emptyset}, \quad g>j>0
$$

(where $c=c_{1}$ ). In our case $c=b_{i}=0$ and so $b_{j, \emptyset}=b_{j,\{1\}}$, and thus $D^{\prime}$ is pulled back from $\bar{M}_{i, 0}$. The same argument applies to the other factor of $\delta_{i}$. Thus by induction on $g$ we may assume $\left.D\right|_{\delta_{i}}$ is nef for any $i>0$ with $b_{i}=0$.

Using $12 \lambda=\kappa+\delta$ and the expression $K_{\bar{M}_{0, n}}=\kappa+\delta$ we have that

$$
f^{*}\left(D / b_{\text {irr }}\right)=K+\sum_{i=1}^{[g / 2]}\left(2-b_{i} / b_{\text {irr }}\right) \delta_{i}+B
$$

where $\delta_{i}$ on $\bar{M}_{0,2 g}$ indicates the pullback of $\delta_{i}$ on $\bar{M}_{g}$ (note the pullback of $\delta_{i}$ to $\bar{R}_{g, n}$ is effective and irreducible) which is an effective sum of boundary divisors with coefficient one (or zero), and $B$ is the sum of the boundary divisors of $\bar{M}_{0,2 g}$ other than the $\delta_{i}$. Let $\Delta=\sum_{i=1}^{[g / 2]}\left(2-b_{i} / b_{\text {irr }}\right) \delta_{i}+B$. By (2.1.4) the coefficients of $\Delta$ are non-negative and, by the above, $K+\Delta$ is nef on any boundary component whose coefficient in $\Delta$ is greater than one. Suppose $D$ (or equivalently $K+\Delta$ ) is not nef. Then there is a $K+\Delta$ negative extremal ray $R$ of $\overline{N E}_{1}\left(\bar{M}_{0,2 g}\right)$. If $\Delta^{\prime}$ is obtained from $\Delta$ by shrinking to any coefficients that are greater than one, then by the above $\left(K+\Delta^{\prime}\right) \cdot R<0$. Thus by KeelMcKernan96, 1.2], $R$ is generated by a one dimensional stratum, which is a contradiction.

Note (6.1) applies to all the edges of the Faber cone computed in Faber96. for $g \leq 7$, giving another proof of $(0.2)$ for $n=0, g \leq 7$. Unfortunately, this fails for $g=10$ where $30 \lambda-3 \delta_{0}-6 \delta_{1}-6 \delta_{2}-2 \delta_{3}-4 \delta_{4}-6 \delta_{5}$ is a vertex. It also answers in the affirmative question (a) at the end of $\S 2$ of [Faber96]:

(6.2) Corollary. (char 0$)$ The ray $10 \lambda-2 \delta+\delta_{\text {irr }}$ is nef on $\bar{M}_{g}$ for all $g \geq 2$.

Also, by averaging with the nef ray $12 \lambda-\delta_{\text {irr }}$, it recovers the ampleness result of CornalbaHarris88]:

(6.3) Corollary (Cornalba-Harris). (char 0) The class $11 \lambda-\delta$ is nef on $\bar{M}_{g}$ for all $g \geq 2$.

The ray $11 \lambda-\delta$ is known to be semi-ample and the associated map to be a birational contraction of relative Picard number one with exceptional locus $\Delta_{1}$, which blows down families of elliptic tails. In fact, except for some special low genus examples, this is the only elementary divisorial blowdown of $\bar{M}_{g}$ :

(6.4) Proposition. (char $0, g \geq 5$ ) The only divisorial contraction $f: \bar{M}_{g, n} \rightarrow X$ of relative Picard number one with $X$ projective is the blowdown of the elliptic tails.

Proof. By (0.9) some boundary divisor is $f$-exceptional. We assume $\delta_{i}$ is exceptional, for some $i \geq 1$; the argument for $\delta_{\text {irr }}$ is only notationally different. By the product decomposition, $f$ induces a fibration on $\bar{M}_{k, 1}$ either for $k=i$ or $k=g-i$. Assume $f$ does not contract the elliptic tails. Then by $(0.9)$, this induced fibration factors through the map $\pi_{k}: \bar{M}_{k, 1} \rightarrow \bar{M}_{k}$. For $i>1$, the general fibers of $\pi_{i}$ and $\pi_{g-i}$ generate the same ray in $N_{1}\left(\bar{M}_{g}\right)$ (indeed, the only basic class with non-zero intersection with either is $\delta_{i}$, which intersects either negatively). Thus for $i>1$, 
$f$ induces a fibration on either factor, and contracts any curve contracted by $\pi_{k}$, $k=i, g-i$. But for $k>2$ (which holds for at least one of $i$ or $g-i$ since $g \geq 5$ ) the image in $N_{1}\left(\bar{M}_{g}\right)$ of $N_{1}\left(\pi_{k}\right)$ (the relative Neron-Severi group of curves of $\pi_{k}$ ) has dimension at least two. Indeed the fiber of $\pi_{k}$ over a general point of $\delta_{1}\left(\bar{M}_{k}\right)$ is a curve with two irreducible components, and a quick computation shows the images in $N_{1}\left(\bar{M}_{g}\right)$ of these components are linearly independent ( $\delta_{1}$ intersects one negatively, and the other positively).

Remark. Bill Rulla [Rulla00 has shown that on $\bar{M}_{3}$ there is a second relative Picard number one contraction of $\delta_{1}$. The corresponding extremal ray is generated by the curve (2.2.4), with $i=1$.

\section{$\S 7$. Reformulation of $F_{1}\left(\bar{M}_{0, n}\right)$ And ReView of the EVidence}

Let $S \subset \bar{M}_{0, n}$ be the union, with reduced structure, of the two dimensional strata. The irreducible components of $S$ are all smooth Del Pezzo surfaces, either $\bar{M}_{0,5}$ or $\mathbb{P}^{1} \times \mathbb{P}^{1}$.

(7.1) Proposition. The natural map $A_{1}(S) \rightarrow A_{1}\left(\bar{M}_{0, n}\right)$ is an isomorphism, and under this map $\overline{N E}_{1}(S)$ is identified with the Faber cone. Thus $F_{1}\left(\bar{M}_{0, n}\right)$ is equivalent to the statement that the natural injection $\overline{N E}_{1}(S) \rightarrow \overline{N E}_{1}\left(\bar{M}_{0, n}\right)$ is an isomorphism.

Proof. The strata generate the Mori cone of $S$, so it's enough to prove the statement about Chow groups. This follows from the Chow group description given in [KontsevichManin96].

Evidence for (0.2). It is known that each stratum of $\bar{M}_{0, n}$ generates an extremal ray. Each can be contracted by a birational morphism of relative Picard number one which is in fact a log Mori fibration (cf. KeelMcKernan96). The main evidence for $F_{1}\left(\bar{M}_{0, n}\right)$ is KeelMcKernan96, 1.2], which states in particular that (0.2) holds for any extremal ray of $\overline{N E}_{1}\left(\bar{M}_{0, n}\right)$ that can be contracted by a morphism of relative Picard number one, except possibly when the map is birational and has one dimensional exceptional locus. Any stratum deforms once $n \geq 9$, so (0.2) would imply this extra condition always holds for such $n$. In practice the main way of showing a curve generates an extremal way is to exhibit a corresponding contraction, so if (0.2) fails, it will be a challenge to find a counter-example.

Kapranov proved that $\bar{M}_{0, n}$ is the inverse limit of all GIT quotients $Q$ (varying the polarization) for $\mathrm{SL}_{2}$ acting diagonally on $\left(\mathbb{P}^{1}\right)^{n}$. In particular each $Q$ is a contraction of $\bar{M}_{0, n}$, and so inherits a stratification. In HuKeel00 $F_{1}(Q)$ and $F^{1}(Q)$ are proved. One can also consider the contractions $\bar{M}_{0, n} \rightarrow \bar{L}_{n-2}$, introduced in [LosevManin00]. $\bar{L}_{n-2}$ is a smooth projective toric variety. On any projective toric variety the toric strata generate the cones of effective cycles. The toric strata for $\bar{L}_{n-2}$ are images of strata in $\bar{M}_{0, n}$, thus $F_{k}\left(\bar{L}_{n-2}\right)$ holds for all $k$. We remark that $\bar{L}_{n-2}$ gives particularly good evidence for $F_{1}\left(\bar{M}_{0, n}\right)$ as it has Picard number $2^{n-2}-n+1$, roughly half the Picard number of $\bar{M}_{0, n}$, whereas a GIT quotient $Q$ can have Picard number at most $n$.

\section{ACKNOWLEDGEMENTS}

We have had interesting and fruitful discussions with various people on topics related to this paper, including X. Chen, F. Cukierman, J. Harris, B. Hassett, 
J. Kollár, A. Logan, J. McKernan, A. Moriwaki, R. Pandharipande, S. Popescu, W. Rulla, S. L. Tan, G. Ziegler, and M. Zou. J. Kollár gave us a counter-example to a theorem claimed in an earlier version of the paper.

Especially inspiring and enlightening were lengthy discussions with Carel Faber. In particular Faber showed us his proofs of $F_{1}\left(\bar{M}_{g}\right), g \leq 4$, and helped us derive the results of $\S 2$.

\section{REFERENCES}

[ArbarelloCornalba96] E. Arbarello and M. Cornalba, Combinatorial and algebro-geometric cohomology classes on the moduli spaces of curves, J. Algebraic Geom. 5 (1996), 705-749. MR 99c:14033

[ArbarelloCornalba98] _ Calculating cohomology groups of moduli spaces of curves via algebraic geometry, Inst. Hautes Études Sci. Publ. Math. 88 (1998), 97-127. MR 2001h:14030

[CHM97] L. Caporaso, J. Harris, and B. Mazur, Uniformity of rational points, J. Amer. Math. Soc. 10 (1997), 1-35. MR 97d:14033

[CornalbaHarris88] M. Cornalba and J. Harris, Divisor classes associated to families of stable varieties, with applications to the moduli space of curves, Ann. Sci. École Norm. Sup. (4) 21 (1988), 455-475. MR 89j:14019

[DeligneMumford69] P. Deligne and D. Mumford, The irreduciblility of the space of curves of given genus, Inst. Hautes Études Sci. Publ. Math. 36 (1969), 75-109. MR 41:6850

[Faber96] C. Faber, Intersection-theoretical computations on $\bar{M}_{g}$, Banach Center Publ. 36, (1996), 71-81. MR 98j:14033

[Faber97] _ Algorithms for computing intersection numbers on moduli spaces of curves, with application to the class of the locus of Jacobians, London Math. Soc. Lecture Note Ser., vol. 264, 1999, pp. 93-109. MR 2000m:14032

[Gibney00] A. Gibney, Fibrations of $\bar{M}_{g, n}, \mathrm{Ph}$. D. Thesis, Univ. of Texas at Austin, 2000 .

[HarrisMorrison98] J. Harris and I. Morrison, Moduli of Curves, Grad. Texts in Math., vol. 187, Springer-Verlag, New York, 1998. MR 99g:14031

[HuKeel00] Y. Hu and S. Keel, Mori dream spaces and GIT, Michigan Math. J. 48 (2000), 331-348. MR 2001i:14059

[Keel92]

$\mathrm{S}$. Keel, Intersection theory of moduli space of stable $N$-pointed curves of genus zero, Trans. Amer. Math. Soc. 330 (1992), 545-574. MR 92f:14003

[Keel99]

[KeelMcKernan96] , Basepoint freeness for nef and big linebundles in positive characteristic, Annals of Math. (1999), 253-286. MR 2000j:14011

[KollarMori98] S. Keel and J. McKernan, Contractible extremal rays of $\bar{M}_{0, n}$, preprint alg-geom/9707016 (1996). collaboration of C. H. Clemens and A. Corti, Cambridge Tracts in Math., vol. 134, Cambridge University Press, Cambridge, 1998. MR 2000b:14018

[KontsevichManin96] M. Kontsevich and Y. Manin, Quantum cohomology of a product. With an appendix by R. Kaufmann, Invent. Math. 124 (1996), 313-339. MR 97e:14064

[Logan00] A. Logan, Relations among divisors on the moduli space of curves with marked points, preprint math.AG/0003104, 2000.

[LosevManin00] A. Losev and Y. Manin, New Moduli Spaces of Pointed Curves and Pencils of Flat Connections, Michigan Math. J. 48 (2000), 443-472. CMP 2001:03

[Moriwaki98] A. Moriwaki, Relative Bogomolov's inequality and the cone of positive divisors on the moduli space of stable curves, J. Amer. Math. Soc. 11 (1998), 569-600. MR 99a:14034

[Moriwaki00] _ Nef divisors in codimension one on the moduli space of stable curves, preprint math.AG/0005012, 2000. 
[Moriwaki01]

[Rulla00] , The Q-Picard group of the moduli space of curves in positive
characteristic, Internat. J. Math. $\mathbf{1 2}(2001)$, no. 5, 519-534.
W. Rulla, Birational Geometry of $\bar{M}_{3}$, Ph D. Thesis, Univ. of Texas at
Austin, 2000.

Department of Mathematics, University of Michigan, Ann Arbor, Michigan 48109

E-mail address: agibney@math.1sa.umich.edu

Department of Mathematics, University of Texas at Austin, Austin, Texas 78712

E-mail address: keel@fireant.ma.utexas.edu

Department of Mathematics, Fordham University, Bronx, New York 10458

E-mail address: morrison@fordham.edu 